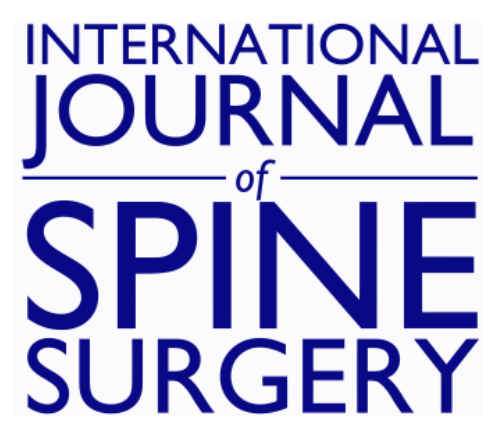

\title{
Effectiveness of Postoperative Wound Drains in One- and Two-Level Cervical Spine Fusions
}

Caroline E. Poorman, Peter G. Passias, Kristina M. Bianco, Anthony Boniello, Sun Yang and Michael C. Gerling

Int J Spine Surg 2014, 8 ()

doi: https://doi.org/10.14444/1034

http://ijssurgery.com/content/8/34

This information is current as of April 26, 2023.

Email Alerts Receive free email-alerts when new articles cite this article. Sign up at: http://ijssurgery.com/alerts 


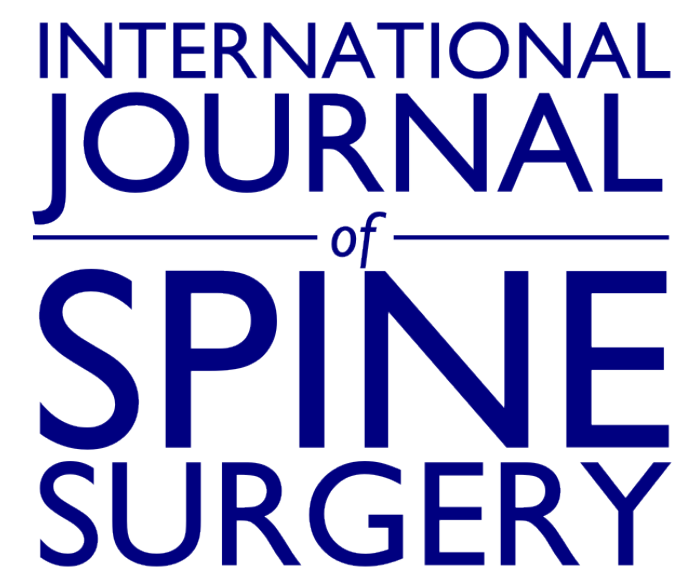

This article generously published free of charge by the International Society for the Advancement of Spine Surgery.

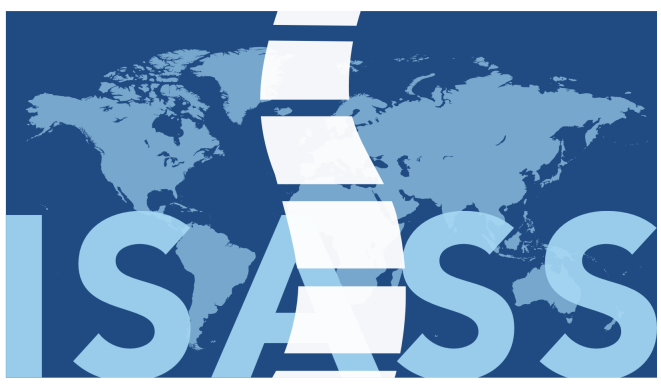

INTERNATIONAL SOCIETY for the ADVANCEMENT of SPINE SURGERY 


\section{Effectiveness of Postoperative Wound Drains in One- and Two- Level Cervical Spine Fusions}

Caroline E. Poorman, BA, Peter G. Passias, MD, Kristina M. Bianco, BA, Anthony Boniello, BS, Sun Yang, BS, Michael C. Gerling, MD

Division of Spinal Surgery, Department of Orthopaedic Surgery, NYU Medical Center Hospital for Joint Diseases, NYU School of Medicine, New York, New York

\section{Abstract}

\section{Background}

Cervical drains have historically been used to avoid postoperative wound and respiratory complications such as excessive edema, hematoma, infection, re-intubation, delayed extubation, or respiratory distress. Recently, some surgeons have ceased using drains because they may prolong hospital stay, operative time, or patient discomfort. The objective of this retrospective case-control series is to investigate the effectiveness of postoperative drains following one- and two-level cervical fusions.

\section{Methods}

A chart review was conducted at a single institution from 2010-2013. Outcome measures included operative time, hospital stay, estimated blood loss and incidence of wound complications (infection, hematoma, edema, and complications with wound healing or evacuation), respiratory complications (delayed extubation, re-intubation, and respiratory treatment), and overall complications (wound complications, respiratory complications, dysphagia, and other complications). Statistical analyses including independent samples ttest, chi-square, analysis of covariance, and linear regression were used to compare patients who received a postoperative drain to those who did not.

\section{Results}

The study population included 39 patients who received a postoperative drain and 42 patients who did not. There were no differences in demographics between the two groups. Patients with drains showed increased operative time (100.1 vs $69.3 \mathrm{~min}, \mathrm{p}<0.001$ ), hospital stay ( 38.9 vs. $31.7 \mathrm{hrs}, \mathrm{p}=0.021)$, and blood loss $(62.7$ vs $29.1 \mathrm{~mL}, \mathrm{p}<0.001)$ compared to patients without drains. The frequency of wound complications, respiratory complications, and overall complications did not vary significantly between groups. 


\section{Conclusions/Level of Evidence}

Cervical drains may not be necessary for patients undergoing one- and two-level cervical fusion. While there were no differences in incidence of complications between groups, patients treated with drains had significantly longer operative time and length of hospital stay.

\section{Clinical relevance}

This could contribute to excessive costs for patients treated with drains, despite the lack of compelling evidence of the advantages of this treatment in the literature and in the current study.

keywords: Cervical wound drain, cervical spine fusion, Spine surgery, postoperative complications

Volume 8 Article 34 doi: 10.14444/1034

\section{Introduction}

Closed wound suction drains have historically been used postoperatively to prevent infections and hematomas. These drains remove blood and fluids from the site of the wound via a drainage tube connected to a drainage bulb that recoils to create a vacuum. The drainage tube is inserted at the time of surgery directly into the wound or through a small separate incision site. The aim of using postoperative closed suction drains is to decrease the likelihood of prolonged drainage from the wound, delayed wound healing, and infection. Closed system drainage is commonly used in a variety of operations including many orthopaedic procedures; however, the efficacy of these drains remains controversial. Although there are many studies in the literature addressing the usage of drains, the wide range of different surgeries in which they are used and studied has yielded mixed results and it is unclear which procedures benefit from postoperative drainage.

Postoperative drains remove blood and debris from the surgical site and theoretically prevent hematoma formation, reducing the risk of infection and wound breakdown. ${ }^{1}$ However, it is possible that they become contaminated and act as a conduit for infection from the exterior into the depths of the wound. ${ }^{2}$ Drains may also be counterproductive by preventing tamponade, resulting in greater postoperative blood loss and increased necessity for blood transfusion. ${ }^{3-5}$ Furthermore, drains may be difficult to remove and in some cases even require additional surgery for removal, particularly if they have been incorrectly placed or sutured to surrounding tissues. Drains may also be displaced or forcibly removed by confused patients, resulting in additional trauma. ${ }^{6}$

Finally, drains may add additional costs to the procedure and increase length of hospital stay. A meta-analysis of several randomized trials by Parker et al. examined total hip and knee replacements, unicompartmental knee replacement, shoulder surgery, iliac crest bone graft donor site, lumbar laminectomy, extensive lumbar spine surgery, spinal scoliosis surgery, anterior cruciate ligament reconstruction, open meniscectomy, hip fracture surgery, and non-emergency fracture fixation or bone grafting and concluded 
there is insufficient evidence to support the routine use of closed suction drainage in these orthopaedic procedures. ${ }^{6}$ Further, this meta-analysis found that the use of wound drains did not result in a decrease in the incidence of wound infections or hematomas.

Additional randomized studies have found that postoperative drains are not effective in preventing infections and epidural hematomas after lumbar spine surgery. ${ }^{1,7}$ However, a study on the use of closed wound drains following posterior spinal fusion for patients with adolescent idiopathic scoliosis by Blank et al. conflicted this finding, reporting decreased wound complications with the use of drains without increased blood loss or need for transfusion. ${ }^{8}$ Thus, the evidence concerning the efficacy of closed suction wound drains following spine surgery remains unknown. To the best of our knowledge, there have not been any studies identifying the efficacy of closed suction wound drains following cervical spine surgery.

In the setting of cervical spine surgery, postoperative drains have traditionally been thought to play a critical role due to theoretical incision healing benefits. Given the proximity of the cervical approach to vital respiratory structures, drains have primarily been used with the hope of preventing potentially fatal respiratory obstructions secondary to excessive edema or hematoma formation. Similarly, drains have been thought to decrease the risk of re-intubation, delayed extubation and respiratory distress, which are respiratory complications that could prolong hospital stay and costs and potentially result in death. Additional key adjacent structures include major vasculature, the recurrent laryngeal nerve, and the esophagus. Therefore, drains have been utilized with the hope of preventing vascular and respiratory compromise and dysphagia.

Some surgeons have recently ceased using postoperative drains in select surgeries secondary to concerns that they may increase patients' length of surgery and hospitalization, as well as overall discomfort. This study examines the effectiveness of postoperative cervical drains for patients undergoing one- and two-level anterior cervical spine fusion surgery. Some surgeons at our institution recently ceased using postoperative drains for these surgeries due to concern that they may not be effectively decreasing complication rates. This study is a retrospective case-control series that compares patients who received postoperative closed wound suction drains to patients who did not receive postoperative drains after the change of common practice. The purpose of this study was to determine whether the presence of a postoperative drain impacts the operative time (OT), length of hospital stay (LOS), length of recovery, estimated blood loss (EBL), hospital re-admittance, and incidence of complications following cervical spine surgery.

\section{Materials and Methods}

\section{Study design and inclusion criteria}

A retrospective case-control series was conducted on patients who received cervical spine fusions consecutively at a single institution between 2010 and 2013. Prior to study initiation, Institutional Review Board approval was obtained. The medical record of each

patient was then reviewed. Inclusion criteria were patients over 18 years of age at the time of surgery who underwent elective one- or two-level anterior cervical spine fusion for radiculopathy and/or myelopathy. Operations for tumor or trauma indications, full 
corpectomies, and revision procedures were excluded. All cases that met inclusion criteria were performed by the same surgical team at a single institution. All patients during the first year of the interval studied invariably received cervical wound drains. After June, 2012 all patients invariably did not receive a drain.

Standard surgical technique was employed using the Smith-Robinson approach from the right side in all patients. ${ }^{9}$ For procedures where drains were utilized, a separate skin incision was made inferior to the wound prior to closure with an 11 blade scalpel, and the drain was tunneled to place the perforated end in the prevertebral space at the operated levels. Drains were placed on suction immediately following closure of the fascial layers. A \#7 Jackson-Pratt was used in all drain cases, except for one patient who received a Penrose drain. The study design was a retrospective case-control series and the analysis compared patients who received a postoperative wound drain to those who did not.

\section{Data collection and analysis}

Demographic measures, such as age, sex, height, weight, and BMI, were collected for all study patients. Peri-operative data collected include American Society of Anesthesiologists (ASA) grade and number of surgical levels. OT, measured from incision to closure, was also recorded. Additional outcomes measures included LOS, measured in hours from hospital admittance to hospital discharge, time of recovery in the post-anesthesia care unit (PACU), estimated blood loss (EBL), hospital re-admittance, and incidence of wound, respiratory, and overall complications, as reported by hospital inpatient charts. Wound complications were defined as documented infection, hematoma, edema, prolonged drainage, wound dehiscence, delayed healing or required evacuation. Respiratory complications were defined as delayed extubation, re-intubation, and respiratory treatment. Overall complications that were assessed included wound complications, respiratory complications, and medical complications, including dysphagia, or any other event that prolonged hospital stay.

\section{Statistical Methods}

All data were analyzed using SPSS software (SPSS, Chicago, Illinois). Statistical tests included independent sample t-tests for continuous variables, and chi-square analysis for comparing frequencies. Analysis of covariance (ANCOVA) and linear regression tests were used to further analyze OT and LOS. Statistical significance was defined by $\mathrm{p}<0.05$. Prior to study initiation, a power analysis revealed that a total sample size of 74 patients, with 37 patients per group, was needed to detect a significant difference in LOS between the two groups (Power $=80 \%$ ).

\section{Results}

A total of 81 patients met inclusion criteria. The study population included 39 patients who received a postoperative drain $(48.1 \%)$ and 42 patients who did not $(51.9 \%)$. There were no significant differences in demographics, including age, sex (percent female), height, weight, BMI, ASA grade, or number of levels fused between the two groups (Table 1). 
Table 1. Demographic variables, ASA grade, and number of levels fused for each study group.

\begin{tabular}{|l|l|l|l|}
\hline Demographics & $\begin{array}{l}\text { Drain } \\
(\mathrm{n}=39)\end{array}$ & $\begin{array}{l}\text { No Drain } \\
(\mathrm{n}=42)\end{array}$ & p-value \\
\hline Age & $46.4 \pm 9.5$ & $45.1 \pm 10.5$ & 0.56 \\
\hline Sex (\% Female) & $53.8 \%$ & $42.8 \%$ & 0.32 \\
\hline Height (cm) & $169.4 \pm 10.8$ & $169.0 \pm 10.6$ & 0.89 \\
\hline Weight (kg) & $79.4 \pm 19.5$ & $80.0 \pm 17.2$ & 0.90 \\
\hline BMI & $27.5 \pm 4.8$ & $27.5 \pm 4.4$ & 0.95 \\
\hline ASA grade & & & 0.36 \\
\hline 1 & $5.1 \%$ & $14.2 \%$ & \\
\hline Two Levels & $79.5 \%$ & $69.0 \%$ & \\
\hline Number of Levels Fused: & & & \\
\hline One Level & $53.8 \%$ & $40.5 \%$ & \\
\hline & & & \\
\hline & & & \\
\hline & & & \\
\hline & & & \\
\hline
\end{tabular}

\section{Operative Duration and Hospital Course}

Analysis revealed that patients with drains had significantly longer OT (100.1 vs. 69.3 min, $\mathrm{p}<0.001)$ and significantly longer LOS (38.9 vs. $31.7 \mathrm{hrs}, \mathrm{p}=0.021)$ than patients without drains. There was no significant difference in length of recovery time in the PACU (246.4 vs. $237.0 \mathrm{~min}, \mathrm{p}=0.72$ ). The average time until drain removal was $24.0 \pm$ 6.7 hours for the drain group; however, this was not recorded in the medical records of one patient and therefore not included. Patients with drains had a significantly higher EBL during surgery than patients without drains (62.7 vs. $29.1 \mathrm{~mL}, \mathrm{p}<0.001$; Table 2).

Table 2. OT, length of recovery in the PACU, LOS, and EBL for each study group.

\begin{tabular}{|l|l|l|l|}
\hline Outcomes & $\begin{array}{l}\text { Drain } \\
(\mathrm{n}=39)\end{array}$ & $\begin{array}{l}\text { No Drain } \\
(\mathrm{n}=42)\end{array}$ & p-value \\
\hline Operative time (min) & $100.1 \pm 36.4$ & $69.3 \pm 19.6$ & $<0.001$ \\
\hline Length of recovery (min) & $246.4 \pm 129.1$ & $237.0 \pm 98.4$ & 0.72 \\
\hline Length of stay (hrs) & $38.9 \pm 16.4$ & $31.7 \pm 10.5$ & 0.021 \\
\hline Estimated Blood Loss (mL) & $62.7 \pm 63.7$ & $29.1 \pm 15.8$ & $<0.001$ \\
\hline
\end{tabular}

A subsequent ANCOVA test adjusting for age, sex, BMI, ASA grade, and number of surgical levels, confirmed a statistically significant difference in OT $(\mathrm{p}<0.001)$, LOS $(\mathrm{p}=0.038)$, and EBL $(\mathrm{p}<0.01)$ between the drain and no-drain groups. ASA grade and number of levels fused were significant variables in the ANCOVA model for OT ( $\mathrm{p}=0.025, \mathrm{p}=0.034$, respectively), with higher ASA grade and higher number of surgical levels corresponding to longer OT. Sex was a significant variable in the ANCOVA model 
for $\operatorname{LOS}(\mathrm{p}=0.046)$, with female patients having a longer LOS than male patients. Further linear regression analysis revealed that $\mathrm{OT}$ and LOS were positively correlated $\left(\mathrm{R}^{2}=0.22\right)$. No patients were re-admitted to the hospital within six weeks of their operation.

\section{Perioperative Complications}

The frequency of overall complications, wound complications, and respiratory complications did not vary significantly between the two groups (Table 3). Overall complications occurred in $41.0 \%$ of the drain group and $45.2 \%$ of the no drain group $(\mathrm{p}=0.70)$. Though this difference was not statistically significant, patients with postoperative drains developed slightly less overall complications than patients without drains $(\mathrm{OR}=0.84)$. Wound complications occurred in $7.5 \%$ of patients in the drain group and $7.1 \%$ of patients in the no drain group $(\mathrm{p}=0.93)$. Patients with drains developed slightly more wound complications $(\mathrm{OR}=1.08)$ than patients without drains. In the drain group, there was a trend towards more complications with wound healing $(5.1 \% \mathrm{vs.} 0.0 \%$, $\mathrm{p}=0.14)$, however, there was less infection $(0.0 \%$ vs. $2.4 \%, \mathrm{p}=0.33)$ and edema $(2.6 \%$ vs $4.8 \%, \mathrm{p}=0.60$ ), although these differences were not significant. Hematoma and wound evacuation did not occur in any patients in either group. Respiratory complications occurred in $17.9 \%$ of the drain group and $16.7 \%$ of the no drain group $(\mathrm{p}=0.88)$. Patients with drains trended towards slightly more respiratory complications that patients without drains $(\mathrm{OR}=1.09)$. The drain group trended towards higher rates of re-intubation $(5.1 \% \mathrm{vs}$. $0.0 \%, \mathrm{p}=0.14)$ and respiratory treatment $(17.9 \%$ vs. $16.7 \%, \mathrm{p}=0.88)$ than the no-drain group. Delayed extubation did not occur in any of the study patients. Finally, dysphagia $(15.4 \%$ vs. $9.5 \%, \mathrm{p}=0.42)$ and all other complications $(30.8 \%$ vs. $26.2 \%, \mathrm{p}=0.65)$ were more frequent in the drain group, although these differences were not statistically significant (Figure 1).

Table 3. Overall complications were defined as having any wound complication, respiratory complication, dysphagia, or other complications.

\begin{tabular}{|l|l|l|l|}
\hline Complications & $\begin{array}{l}\text { Drain } \\
(\mathrm{n}=39)\end{array}$ & $\begin{array}{l}\text { No drain } \\
(\mathrm{n}=42)\end{array}$ & $\mathrm{p}$-value \\
\hline Overall Complications & $41.0 \%$ & $45.2 \%$ & 0.70 \\
\hline Wound Complications & $7.7 \%$ & $7.1 \%$ & 0.93 \\
\hline Wound healing & $5.1 \%$ & $0.0 \%$ & 0.14 \\
\hline Infection & $0.0 \%$ & $2.4 \%$ & 0.33 \\
\hline Wound evacuation & $0.0 \%$ & $0.0 \%$ & - \\
\hline Hematoma & $0.0 \%$ & $0.0 \%$ & - \\
\hline Edema & $2.6 \%$ & $4.8 \%$ & 0.6 \\
\hline Respiratory complications & $17.9 \%$ & $16.7 \%$ & 0.88 \\
\hline Reintubation & $5.1 \%$ & $0.0 \%$ & 0.14 \\
\hline Delayed extubation & $0.0 \%$ & $0.0 \%$ & - \\
\hline Despiratory treatment & $17.9 \%$ & $16.7 \%$ & 0.88 \\
\hline & $15.4 \%$ & $9.5 \%$ & 0.42 \\
\hline
\end{tabular}




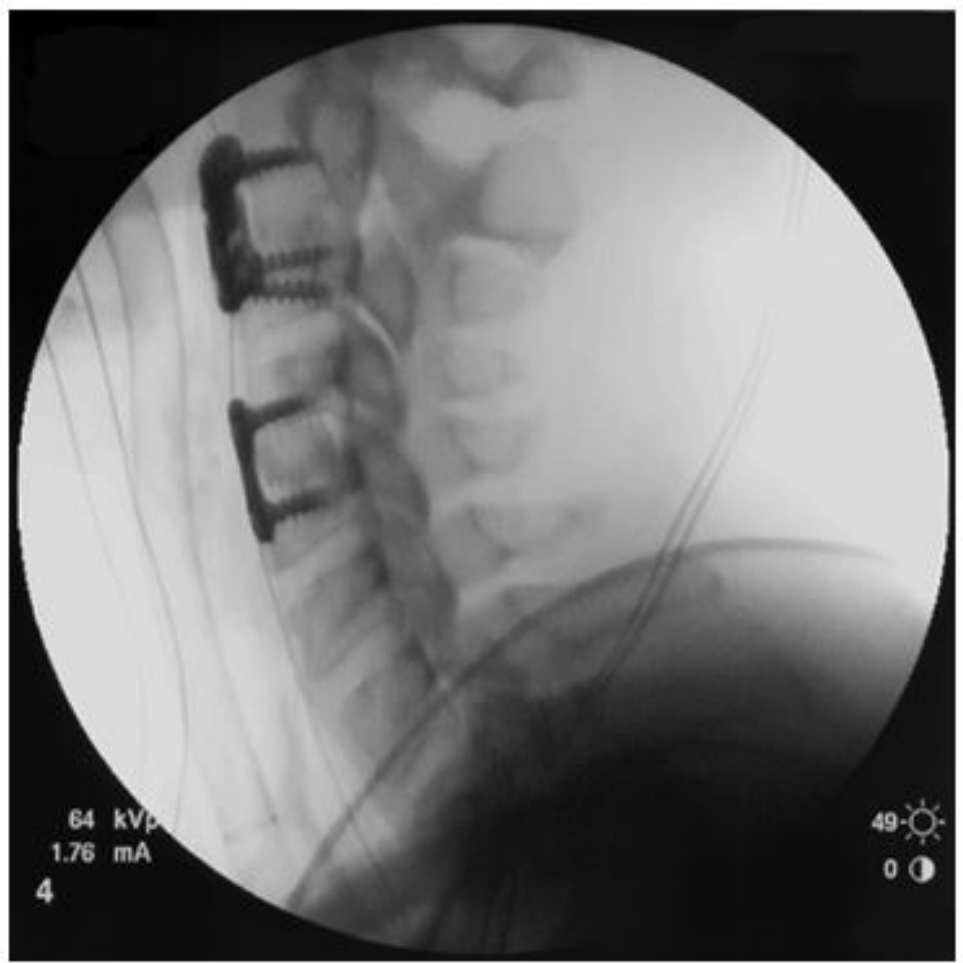

Fig. 1. Intraoperative fluoroscopy performed after a C4-C5 anterior decompression and fusion on a 44 year old female presenting with herniated nucleus pulposus and radiculopathy. Patient received a postoperative JP drain. Minimal peri-incisional edema and sinus bradycardia noted on postoperative day 0 (POD0). On POD1 patient was noted to be complaining of chest pain, hoarseness and difficulty swallowing.

\section{Discussion}

The use of postoperative wound drains in orthopaedic spinal surgery, and specifically cervical surgery, remains controversial. To our knowledge, this is the first series evaluating the utility of drains following cervical spine surgery. Our study revealed that patients with drains had significantly longer OT, longer LOS, and higher EBL than patients without drains. However, the frequency of overall complications, wound complications, and respiratory complications did not vary significantly between the two groups.

The most clinically relevant finding in the current study is the lack of significant difference in the incidence of respiratory complications and treatments between the two groups. Many surgeons seek methods to minimize the risk of respiratory complications in anterior cervical spine surgery, given their potentially fatal consequences. Considering the known complications of edema and hematoma formation in any surgical procedure, it would teleologically make sense to employ wound drains, particularly in this anatomically precarious region. The current study found that although there were no 
differences in respiratory complications between the groups, the drain group showed a higher trend of re-intubation and respiratory treatment. A study by Youssef et al., on the use of drains after carotid endarterectomies also found no statistically significant differences in respiratory complications or distress between patients who received a drain and those who did not. ${ }^{10}$ These findings therefore suggest that employing cervical wound drains does not achieve the primary goal of preventing serious respiratory complications.

Postoperative drains have previously been used in order to prevent wound complications such as hematoma formation and infection. A meta-analysis of general orthopaedic surgeries by Parker et al. found that the occurrence of wound infection and wound hematoma did not differ between patients who received closed suction wound drains and those that did not. ${ }^{6}$ Another study examined single-level lumbar laminectomies, and found that there was no difference in infection rates between patients who received a postoperative wound drain and those who did not. ${ }^{7}$ Furthermore, a randomized study on the use of closed wound suction drains for extensive lumbar spine surgery by Brown et al.found that the use of a postoperative drain did not decrease the risk of infection or hematoma. ${ }^{1}$ Our findings pertaining to the cervical region draw similar conclusions to these lumbar studies, as we found no significant different in rates of infections and hematoma formation. In the setting of a different cervical surgery in the same region, Youssef et al. also found no statistically significant differences difference in the risk for wound hematomas during thyroidectomy and carotid endartectomy procedures. ${ }^{10}$

Similarly, the utility of cervical drains in preventing delayed wound healing has little supporting evidence in the literature. In the setting of single-level lumbar laminectomy, Payne et al. found that there was no difference in wound healing between patients who received a postoperative wound drain and those who did not. ${ }^{7}$ These findings agree with our study, which also did not find a statistically significant difference in delayed healing between the two groups. These studies call into question the usage of drains in a range of different settings, and highlight the need for controlled prospective data regarding postoperative drains.

Our present study found that patients with drains had a significantly increased OT. In contrast, Brown et al. did not find any significant differences in OT between patients who received a postoperative wound drain and those that did not following lumbar spine surgery. ${ }^{1}$ It is possible that our finding of increase in operative time is due to the placement of the drain, which in our institution is done through a separate small incision. However, the retrospective nature of the present study may also contribute to the difference in OT because the drain group was drawn from 2010-June 2012 while the nodrain cohort came after June 2012. It is possible that other procedural differences contributed to the length of OT, although these were not identifiable in the chart review. ASA grade and number of surgical levels were significant covariates in the ANCOVA model for OT. Thus, an increase in OT could be due to the patient's ASA grade and the complexity of surgical cases. However, when controlling for these variables, the results of the ANCOVA model confirmed a difference in OT between the drain and no drain groups. Nevertheless, an important factor is that the cervical surgeries included in this study are relatively short procedures, making comparison to the OT of other orthopaedic surgeries difficult. 
In the present study, the average LOS was 38.9 hours for the drain group and 31.7 hours for the no drain group after anterior cervical spine surgery. LOS was found to be significantly increased for the drain group. These findings are inconsistent with the metaanalysis of general orthopaedic procedures by Parker et al., which identified no significant differences in LOS between the drain and no drain groups following various orthopaedic procedures. ${ }^{6}$ Other orthopaedic surgeries also have longer LOS, with studies reporting an average 4.3-18 day stay for total knee and hip arthroplasty ${ }^{11-13}$ and 12.5 for knee prosthesis implantation. ${ }^{14}$ In the setting of lumbar spine surgery, Brown et al. found an average LOS of 3.32 days for the drain group and 3.92 days for the no-drain group, which were not significantly different. ${ }^{1}$

Extending conclusions concerning LOS from lumbar to cervical surgeries is difficult since lumbar surgeries generally require keeping a patient in the hospital longer, due to the complexity of cases. Since cervical surgeries require smaller LOS, a delay of removing a drain will have more of a likelihood of extending the LOS. Therefore, the effect of drains may be more relevant to LOS in the setting of cervical surgical procedures because there is a lower mean duration of LOS, with some patients being discharged on the day of surgery. In a meta-analysis on the use of wound drains following thyroidectomies, patients who had postoperative wound drains showed significantly prolonged LOS, with a mean of 1.18 days for patients with a suction drain. ${ }^{15}$ These findings agree with our current findings regarding surgery in the neck, suggesting cervical wound drains may increase LOS following cervical spine surgery.

In general, longer OT occurred during more complex cases, which in turn, increased LOS. This is confirmed by the results of the linear regression model, which showed a positive correlation between OT and LOS. Since the average time before drain removal was 24.5 hours for the drain group, the difference in LOS between the drain and no drain groups was likely due to the differences in OT and not due strictly to the presence of the drain extending the LOS. Furthermore, differences in LOS could be due to other variables, such as female sex, which was a significant variable in the ANCOVA model. However, despite female patients having a higher LOS than male patients, the differences in LOS between the drain and no drain groups were still statistically different.

Several studies have found that total EBL was greater for patients who received postoperative drains than for patients without postoperative drains in hip and knee replacement surgery and anterior cruciate ligament reconstruction. ${ }^{16-20}$ However, other studies did not find a statistically significant difference in EBL when comparing patients with or without surgical drains. ${ }^{1,14,21}$ Our present study found patients with postoperative surgical drains had significantly higher EBL. Since patients uniformly stopped receiving drains at a certain point in time, a bias for cases with higher EBL to receive drains was eliminated. Therefore, this difference is likely attributed to the fact that patients in the drain group had a longer operative time. Future prospectively randomized studies are needed to further investigate whether wound drains affect operative blood loss following anterior cervical spine surgery.

The main limitation of this study is the retrospective design. Without a randomized, prospective study design, it is impossible to limit confounding factors. In order to mitigate the problems inherent with retrospective studies, all patients included in this study were consecutively treated during the study period of 2010 to 2013. Halfway through the study 
period, the surgeons decided to stop using surgical drains due to concerns that they may increase OT, LOS, and patient discomfort. The fact that the surgeons stopped using drains during the study period may be a limitation to the study because the patient cohort that did not receive surgical drains was more recent than the cohort of patients that did receive drains. Thus, the differences in OT, EBL and LOS could be potentially attributed to differences in hospital practices, data collection, or increased vigilance and surgical performance over time. Conversely, the retrospective nature of the study ensured that all patients prior to a certain time period had surgical drains and all patients after that time period did not have drains, thus eliminating a potential bias for more complicated operations to receive drains.

This present study aims to investigate the use of postoperative wound drains after cervical spine surgery. Our results indicate that the use of postoperative drains result in longer OT and LOS for patients following one- and two-level cervical spine surgeries. Furthermore, we found that the drain group had a larger average EBL during surgery. However, we did not find any statistically significant differences in complication rates, particularly in respiratory complications, between the two groups. Our results suggest that cervical drains may not have a clear role for patients undergoing one- and two-level cervical fusion. While there were no significant differences in incidence of complications between the two groups in this study, the patients treated with postoperative drains had significantly longer OT and LOS. These findings suggest that there may be no benefit in using postoperative drains, and may potentially expose patients to more risks following cervical spine surgeries. In light of the findings and concurrent limitations of this series, future studies are needed to address the lack of compelling evidence supporting the use of cervical wound drains with particular emphasis on adverse effects, peri-operative morbidity, immediate outcomes, and procedure related cost.

\section{References}

1. Brown MD, Brookfield KF. A randomized study of closed wound suction drainage for extensive lumbar spine surgery. Spine. 2004;29(10):1066-1068.

2. Willett K, Simmons C, Bentley G. The effect of suction drains after total hip replacement. Journal of Bone \& Joint Surgery, British Volume. 1988;70(4):607-610.

3. Cobb J. Why use drains. J Bone Joint Surg Br. 1990;72(6):993-995.

4. Hallstrom B, Steele J. Postoperative course after total hip arthroplasty: wound drainage versus no drainage. Orthopaedic review. 1992;21(7):847-851.

5. Reilly T, Gradisar Jr IA, Pakan W, Reilly M. The use of postoperative suction drainage in total knee arthroplasty. Clinical orthopaedics and related research. 1986;208:238-242.

6. Parker M, Livingstone V, Clifton R, McKee A. Closed suction surgical wound drainage after orthopaedic surgery. Cochrane Database Syst Rev. 2007;3.

7. Payne DH, Fischgrund JS, Herkowitz HN, Barry RL, Kurz LT, Montgomery DM. Efficacy of closed wound suction drainage after single-level lumbar laminectomy. Journal of Spinal Disorders \& Techniques. 1996;9(5):401-403.

8. Blank J, Flynn JM, Bronson W, et al. The use of postoperative subcutaneous closed suction drainage after posterior spinal fusion in adolescents with idiopathic scoliosis. Journal of Spinal Disorders \& Techniques. 2003;16(6):508-512. 
9. Cheung KM, Mak KC, Luk KD. Anterior approach to cervical spine. Spine (Phila Pa 1976). Mar 1 2012;37(5):E297-302.

10. Youssef F, Jenkins M, Dawson K, Berger L, Myint F, Hamilton G. The value of suction wound drain after carotid and femoral artery surgery: a randomised trial using duplex assessment of the volume of post-operative haematoma. European journal of vascular and endovascular surgery. 2005;29(2):162-166.

11. Holt BT, Parks NL, Engh GA, Lawrence JM. Comparison of closed-suction drainage and no drainage after primary total knee arthroplasty. Discussion. Orthopedics. 1997;20(12):1121-1125.

12. Walmsley P, Kelly M, Hill R, Brenkel I. A prospective, randomised, controlled trial of the use of drains in total hip arthroplasty. Journal of Bone \& Joint Surgery, British Volume. 2005;87(10):1397-1401.

13. Crevoisier X, Reber $P$, Noesberger B. Is suction drainage necessary after total joint arthroplasty? Archives of orthopaedic and trauma surgery. 1998;117(3):121-124.

14. Jenny J-Y, Boeri C, Lafare S. No drainage does not increase complication risk after total knee prosthesis implantation: a prospective, comparative, randomized study. Knee Surgery, Sports Traumatology, Arthroscopy. 2001;9(5):299-301.

15. Samraj K, Gurusamy K. Wound drains following thyroid surgery. Cochrane Database Syst Rev. 2007;4.

16. Murphy J, Scott J. The effectiveness of suction drainage in total hip arthroplasty. Journal of the Royal Society of Medicine. 1993;86(7):388.

17. Widman J, Jacobsson H, Larsson SA, Isacson J. No effect of drains on the postoperative hematoma volume in hip replacement surgery: a randomized study using scintigraphy. Acta Orthopaedica. 2002;73(6):625-629.

18. Esler C, Blakeway C, Fiddian N. The use of a closed-suction drain in total knee arthroplasty. A prospective, randomised study. J Bone Joint Surg Br. 2003;85(2):215-217.

19. Mengal B, Aebi J, Rodriguez A, Lemaire R. A prospective randomized study of wound drainage versus non-drainage in primary total hip or knee arthroplasty]. Revue de chirurgie orthopédique et réparatrice de l'appareil moteur. 2001;87(1):29.

20. Paessler $\mathrm{H}$, Buchgraber A. Is wound drainage in ACL reconstructive surgery necessary? A prospective and randomized study. Orthop Trans. 1997;21(4):1233-1234.

21. Johansson T, Engquist M, Pettersson L-G, Lisander B. Blood loss after total hip replacement: a prospective randomized study between wound compression and drainage. The Journal of arthroplasty. 2005;20(8):967-971.

\section{Corresponding Author}

Peter G. Passias, New York Spine Institute, 361 Merrick Avenue, Westbury, New York 11590, peter.passias@nyumc.org.

\section{Disclosures}

Michael C. Gerling, MD is currently receiving honoraria from Striker for consulting and lectures. However, this is not related to the present research. For the remaining authors, no conflicts of interest and source funding were declared. 
Copyright (C) 2014 ISASS - International Society for the Advancement of Spine Surgery.

To see more or order reprints or permissions, see http://ijssurgery.com. 